\title{
Spin polarization and transition from metallic to insulating behavior in $2 \mathrm{D}$ systems
}

\author{
E. Tutuc, E.P. De Poortere, S.J. Papadakis and M. Shayegan \\ Department of Electrical Engineering, Princeton University, Princeton, NJ 08544
}

\begin{abstract}
We have made quantitative measurements of the spin polarization of two-dimensional (2D) GaAs (100) electrons and GaAs (311)A holes, as a function of an in-plane magnetic field. The functional form of the in-plane magnetoresistance is shown to be intimately related to the spin polarization. Moreover, for three different 2D systems, namely GaAs (100) electrons, GaAs (311)A holes, and AlAs (411)B electrons, the temperature dependence of the in-plane magnetoresistance reveals that their behavior turns from metallic to insulating before they are fully spin polarized.
\end{abstract}

PACS numbers: 73.50.-h, 71.30.+h, 71.70.Ej

A 2D carrier system, long believed to have an insulating ground state [1], 2], displays a metallic behavior at finite temperature in certain ranges of densities [3]. The response of the $2 \mathrm{D}$ system to a parallel magnetic field $(B)$ may help understand this unexpected phenomenon [1, 5, 6, 7, 8, 9, 10, 11, 12, 13): when $B_{\|}$is increased above a certain threshold value, $B_{I}$, the behavior of the $2 \mathrm{D}$ system changes to insulating. Another effect of $B_{\|}$is, of course, to create a Zeeman splitting between the spinup and spin-down states. This induces a partial spin polarization of the system so that two spin subbands with different populations are formed. When $B_{\|}$is increased above a characteristic field $B_{P}$, the $2 \mathrm{D}$ system becomes fully spin polarized. The main goal of our work is to experimentally explore the relation between $B_{I}$ and $B_{P}$.

Here we report measurements of the fields $B_{P}$ and $B_{I}$ in three different 2D systems. We first present, via Shubnikov - de Haas (SdH) measurements in a nearly parallel field [7], a quantitative determination of the spin polarization of a dilute GaAs 2D electron system as a function of $B_{\|}$. The data allow us to find $B_{P}$. We then show that the sample magnetoresistance (MR), measured as a function of a purely parallel field, reveals a clear break at $B_{P}$, therefore providing an alternative method for determining $B_{P}$. Our previous results, detailed elsewhere [7], showed a very similar trend for GaAs 2D holes. We also present the temperature $(T)$ dependence of the inplane MR to determine the field $B_{I}$ for three different modulation-doped 2D systems: GaAs electrons, GaAs holes, and AlAs electrons. In all three systems, $B_{I}$ is well below $B_{P}$ implying that the transition to insulating behavior always occurs well before the full spin-polarization of the system.

Figures 1(a) and (b) illustrate how we use SdH measurements to determine the spin polarization. As shown schematically in Fig. 1(a), application of a $B_{\|}$to the 2D system separates the two spin subbands by the Zeeman energy, $E_{Z}$. In our experiments [Fig. 1(b)] we apply a constant magnetic field $(B)$, slowly rotate the sample around $\theta=0^{\circ}$ to introduce a small $B_{\perp}$, and measure the sample resistance during the rotation. We then Fourier analyze [14] the $\mathrm{SdH}$ oscillations induced by $B_{\perp}$, as their frequencies directly give the two spin subbands' densities. We emphasize that in our measurements we are able to limit $B_{\perp}$ to sufficiently small values so that $B_{\|} \cong B$ to better than $2 \%$ during the sample rotation.

In Fig. 1 (c) we show plots of resistance $R$ vs $B_{\perp}$, all taken for a GaAs (100) 2D electron system at a fixed total density, $n=2.05 \times 10^{10} \mathrm{~cm}^{-2}$ [15]. The trace in top box was taken with $\theta$ fixed at $90^{\circ}$ and represents standard $\mathrm{SdH}$ measurements, i.e., $B_{\|}=0 \mathrm{~T}$. The Fourier transform of $R$ vs $1 / B_{\perp}$, shown in the top box in Fig. 1(d), exhibits two peaks, one at $0.85 \mathrm{~T}$ and another at half this value, at $0.42 \mathrm{~T}$. The $0.85 \mathrm{~T}$ peak, multiplied by $(e / h)$, gives the total density of the $2 \mathrm{D}$ electron system. The peak at $0.42 \mathrm{~T}$ stems from the spin-unresolved oscillations. The rest of the traces shown in Fig. 1(c) were taken by rotating the sample at the indicated constant $B$ applied almost parallel to the 2D plane. With increasing $B_{\|}$, we observe a splitting of the lower Fourier transform peak $(0.42 \mathrm{~T})$ into two peaks. The frequencies of these two peaks, multiplied by $(e / h)$, give the two spin subband populations. We note that the two populations add up to the total density of the sample. As $B_{\|}$is increased, the majority spin subband peak merges with the total density peak $(0.85 \mathrm{~T})$ and the minority spin subband peak moves to very low frequencies and is no longer resolved [16].

In Fig. 2 we summarize the positions of the Fourier transform peaks corresponding to the majority and minority spin subbands as a function of $B_{\|}$for the case examined in Fig. 1. Above a certain field $B_{P}$, the majority spin subband population saturates at a value which corresponds to the total $2 \mathrm{D}$ density. Therefore, $B_{P}$ marks the onset of full spin polarization. We also measured the inplane MR, by fixing $\theta$ at $0^{\circ}$ and recording the resistance as a function of a purely parallel magnetic field. The MR trace, taken for $n=2.05 \times 10^{10} \mathrm{~cm}^{-2}$, is also shown in Fig. 2. This trace exhibits a clear break in the functional form of the MR as it changes from a strong $\sim e^{B^{2}}$ dependence at low field to a weak $\sim e^{B}$ dependence at higher fields. The data in Fig. 2 demonstrate that the break, i.e., the onset of simple exponential behavior of the inplane MR, coincides with the field $B_{P}$ above which the spins are fully polarized. Remarkably, GaAs $2 \mathrm{D}$ holes exhibit a similar functional form for the in-plane MR, and the field of full spin polarization also coincides with the onset of the exponential behavior 5 , 6, 67.

Of particular relevance to the anomalous metallic be- 
(a)
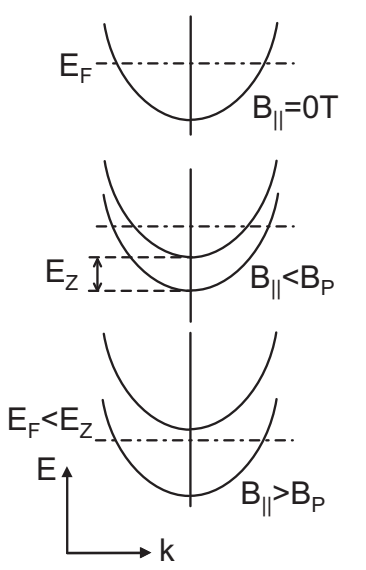

(b)

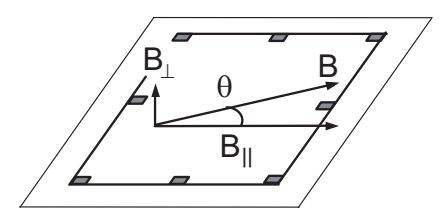

(c)

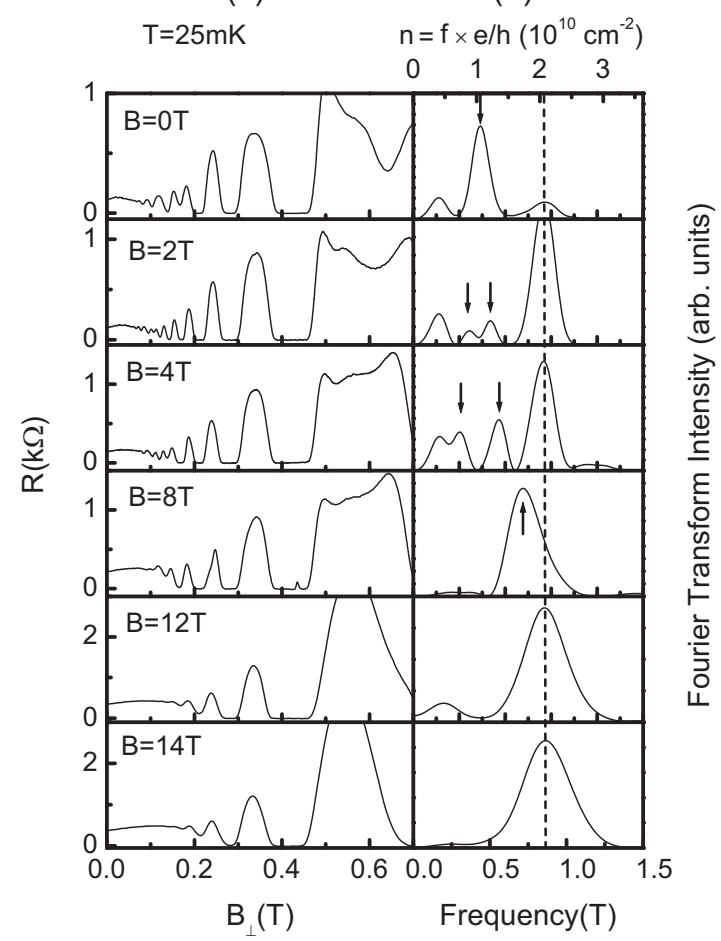

FIG. 1: (a) Sketches of energy vs k-vector illustrating the evolution of spin subbands with increasing parallel field. (b) Experimental geometry. (c) Resistance vs $B_{\perp}$, for a GaAs (100) $2 \mathrm{D}$ electron system with total density $n=2.05 \times 10^{10} \mathrm{~cm}^{-2}$, at the indicated parallel fields. (d) Fourier transforms of the traces in (c).

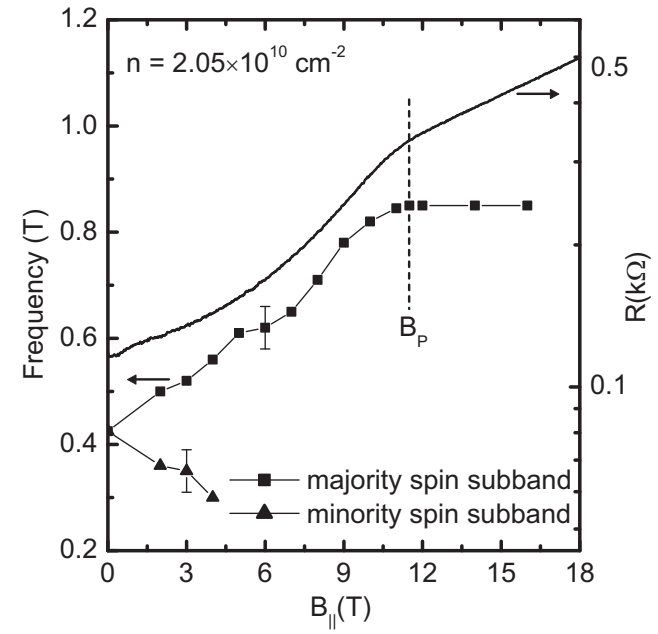

FIG. 2: Summary of Fourier transform peak positions of Fig. $1(d)$, as a function of applied magnetic field, along with the in-plane magnetoresistance trace. The dashed vertical line marks the field $B_{P}$ above which the $2 \mathrm{D}$ electrons becomes fully spin polarized.

havior in 2D systems is the temperature dependence of the in-plane MR. In Fig. 3 we show examples of such data for three different 2D systems: GaAs (100) electrons, GaAs (311)A holes and AlAs (411)B electrons 17 . The data, in agreement with previous results 地, 5, 6, reveal that the metallic behavior observed at zero magnetic field turns insulating above a certain field, $B_{I}$. The data also clearly show that $B_{I}$ is always smaller than $B_{P}$, i.e., the transition to the insulating phase occurs before the $2 \mathrm{D}$ system is fully spin polarized.

These observations provide strong experimental support for a link between the presence of two subbands with finite populations and the metallic behavior. We suggest that a temperature dependent intersubband scattering mechanism may be responsible for the metallic behavior.

To summarize, we have measured the spin subband populations of different $2 \mathrm{D}$ systems as a function of a parallel magnetic field. We establish a direct connection between the functional form of the in-plane MR and the spin polarization of the $2 \mathrm{D}$ system. Temperature dependence of in-plane MR data for three distinct 2D systems clearly shows that each 2D system turns insulating before it is fully spin polarized.

Our work was supported by ARO, DOE, NSF, and the von Humboldt Foundation. We thank R. Winkler for valuable discussions. 

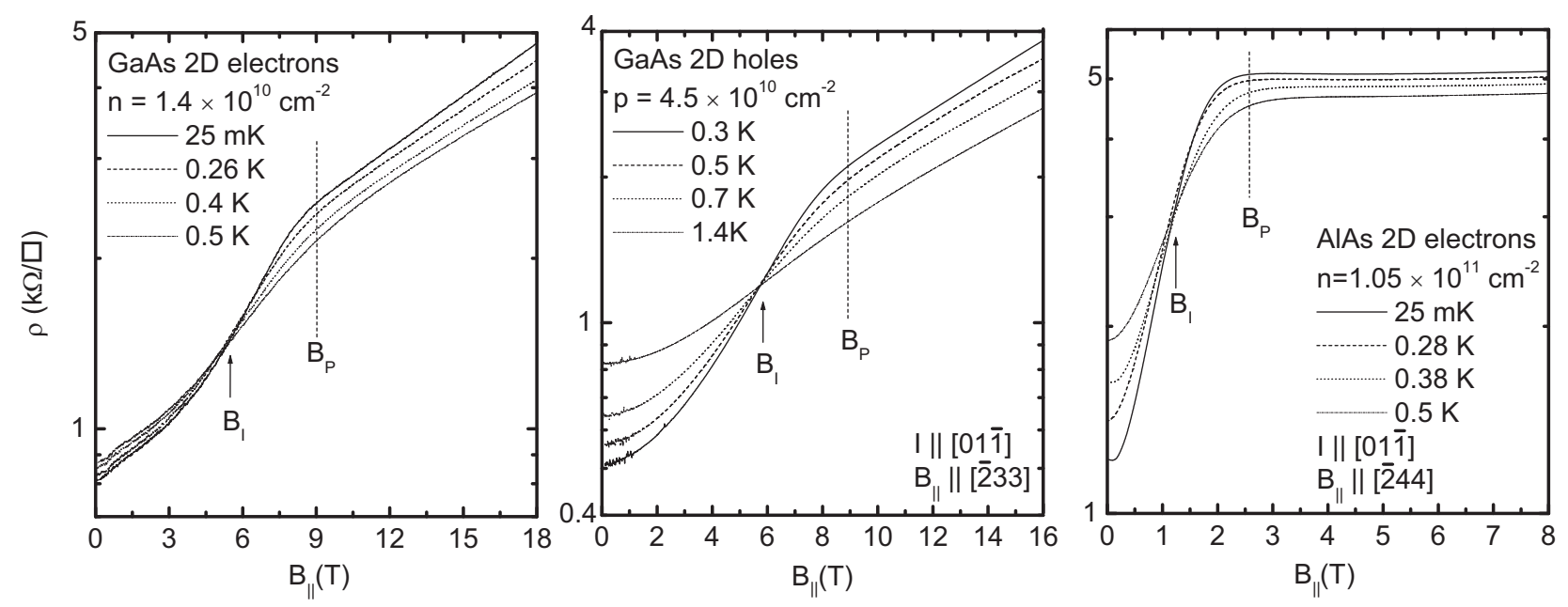

FIG. 3: Temperature dependence of the in-plane MR for three different 2D systems: (a) GaAs (100) electrons (b) GaAs (311)A holes and (c) AlAs (411)B electrons. The density of each 2D system is indicated in the respective panel. The fields $B_{I}$ and $B_{P}$ mark the onsets of insulating behavior and full spin polarization, respectively.

[1] E. Abrahams et al., Phys. Rev. Lett. 42, 673 (1979).

[2] D. Bishop et al., Phys. Rev. Lett. 44, 1153 (1980).

[3] S.V. Kravchenko et al., Phys. Rev. Lett. 77, 4938 (1996); D. Popović et al., Phys. Rev. Lett. 79, 1543 (1997); P.T. Coleridge et al., Phys. Rev. B 56, R12764 (1997); Y. Hanein et al., Phys. Rev. Lett. 80, 1288 (1998); M.Y. Simmons et al., Phys. Rev. Lett. 80, 1292 (1998); S.J. Papadakis and M. Shayegan, Phys. Rev. B 57, R15068 (1998).

[4] K. Mertes et al., Phys. Rev. B 60, 5093 (1999).

[5] J. Yoon et al., Phys. Rev. Lett. 84, 4421 (2000).

[6] S.J. Papadakis et al., Phys. Rev. Lett. 84, 5592 (2000).

[7] E. Tutuc et al., Phys. Rev. Lett. 86, 2858 (2001).

[8] T. Okamoto et al., Phys. Rev. Lett. 82, 3875 (1999).

[9] S.A. Vitkalov et al., Phys. Rev. Lett. 85, 2165 (2000); S.A. Vitkalov et al., cond-mat/0009454.

[10] S. Murzin et al., JETP Lett. 67, 113 (1999).

[11] S.J. Papadakis et al., Science 283, 2056 (1999).

[12] Y. Yaish et al., Phys. Rev. Lett. 84, 4954 (2000).

[13] S.J. Papadakis et al., Phys. Rev. B 62, 15375 (2000).
[14] In the range of magnetic field where the resistance displays $\mathrm{SdH}$ oscilations we first plot $R$ vs $1 / B_{\perp}$. We then substract a fitted linear background to center the oscillations around zero, multiply the signal with a Hamming window, and then use a fast Fourier transfrom algorithm.

[15] The total density was determined from the positions of integer and fractional quantum Hall states developed at fields higher than $0.5 \mathrm{~T}$.

[16] The peaks observed at very low frequencies $(<0.2 \mathrm{~T})$ in Fig. 1(d) are Fourier transform artifacts.

[17] Data shown in the right panel of Fig. 3 are from 2D electrons confined to an AlAs quantum well which is flanked by Si-modulation-doped $\mathrm{Al}_{0.4} \mathrm{Ga}_{0.6}$ As barriers. The $2 \mathrm{D}$ electrons in such a system occupy the conduction-band minima at the $\mathrm{X}$ points of the Brillouin Zone. For more details, see S.J. Papadakis and M. Shayegan, Phys. Rev. B 57, R15068 (1998), and E.P. De Poortere et al., Science 290, 1546 (2000). 